\title{
Feticismo e narcisismo - la base del capitalismo?
}

Anselm Jappe ${ }^{4}$

"Feticismo" e "narcisismo" sono due termini che si sentono spesso nei discorsi quotidiani, soprattutto negli ultimi tempi. A prima vista, il loro significato sembra ben chiaro. II narcisismo è l'atteggiamento di colui o colei che si guarda in continuazione allo specchio e esalta il proprio aspetto esteriore, e più in generale indica una persona egoista, troppo convinta dei propri pregi, che vuole far girare il mondo, e soprattutto la vita degli altri, intorno a sé. Diventa allora un pericolo sul luogo di lavoro, soprattutto se si tratta di un capo, ed è una disgrazia averla come partner in una relazione. Secondo un'affermazione corrente, le reti sociali hanno aumentato fortemente le tendenze narcisistiche nella società.

II feticismo invece indica l'amore ritenuto eccessivo per qualcosa (un oggetto, un comportamento, una persona). Possiamo allora trovare, per esempio, un feticista della musica di Rossini, oppure, con riferimento al feticismo sessuale di cui parla Sigmund Freud, un uomo che si eccita vedendo delle scarpe a tacchi alti o il cuoio. Spesso viene fatto riferimento al "feticismo della merce" introdotto da Karl Marx nel dibattito, e ci si riferisce allora, normalmente per condannarli, a coloro che stravedono per le grosse macchine o per una marca di vestiti il cui consumo, si sospetta, deve nascondere la povertà delle loro vite.

Questi usi delle parole feticismo e narcisismo non sono falsi: essi coprono dei fenomeni ben reali e che si incontrano tutti i giorni. E la questione non è se rimangano fedeli alle definizioni originarie date da Freud, da Marx o, nel caso del feticismo, anche dalla storia delle religioni

$4 \quad$ Academia delle Belle Arti de Sassari. 
e dall'antropologia. Ma si tratta qui di comprendere se questi due concetti non permettano un uso più vasto e più profondo che arriva fino al cuore della società contemporanea - un uso che deriverebbe da una lettura rinnovata dei concetti originali di Max e di Freud, senza però fissarsi sempre sulla loro lettera.

Il feticismo della merce viene introdotto da Marx alla fine del primo capitolo del Capitale (1867), dopo aver analizzato le categorie basilari della società produttrice di merci, e dunque del capitalismo: il lato astratto del lavoro, detto lavoro astratto, cioè la semplice erogazione di energia umana indifferenziata, misurata in tempo, che forma il valore delle merci (materiali o immateriali) il quale si rappresenta finalmente nel denaro. Marx descrive il feticismo come un rapporto sociale tra le cose e un rapporto di cose tra le persone, espressione di un modo di produzione dove la produzione dirige l'uomo invece che l'uomo la produzione. Gli uomini mettono in rapporto i loro lavori privati non direttamente, ma soltanto in una forma oggettivata, sotto un'apparenza di cose, cioè come quantità determinate di lavoro umano uguale, espresso nel valore di una merce. Tuttavia, non sanno di farlo e attribuiscono i movimenti dei loro prodotti - gli scambi tra i produttori e le proporzioni in cui scambiano le merci - a qualità naturali di questi ultimi. Il feticismo è un processo incosciente e collettivo che nasconde la vera natura della produzione capitalista. Marx stesso lo qualifica di "misterioso" e ricorre a formule come quella secondo cui la merce è "sensibile- ,sovrasensibile" ed è comparabile alla religione dove l'uomo proietta le sue forze su un essere trascendente da cui crede poi di dipendere.

Queste pagine di Marx - che hanno degli echi anche in alcuni altri passaggi delle sue opere - sono state o trascurate dalla maggior parte degli interpreti di Marx oppure sono state comprese come la denuncia di una mistificazione che ha perennemente luogo nella società capitalista: il feticismo naturalizza - cioè presenta falsamente come naturale le condizioni di sfruttamento e di furto del pluslavoro (e dunque del plusvalore) a danno degli operai che caratterizza il capitalismo. Questo travestimento della realtà capitalista esiste evidentemente. Ma il feticismo nel senso originario marxiano va ben oltre. Non è solo un fenomeno ideologico, appartenente alla sfera della coscienza, o addirittura un inganno consapevole da parte degli economisti borghesi che vela la 
realtà quotidiana dello sfruttamento. La forza del concetto di Marx sta nel fatto di mettere a nudo l'inversione reale che avviene nel capitalismo. II lato astratto del lavoro (erogazione di lavoro come pura quantità e senza riguardo per il contenuto) prevale sul suo lato concreto, il valore mercantile prevale sul valore d'uso. Conta solo la quantità di lavoro svolto e la parte di pluslavoro che contiene, perché si traduce in valore, in plusvalore e finalmente in profitto. Che cosa viene prodotto da questo lavoro, è secondario o irrilevante. Conta solo il suo accrescimento quantitativo, la sua accumulazione. Se si producono bombe oppure grano importa ben poco dal punto di vista del valore: si produrranno più bombe e meno grano se le bombe contengono più plusvalore. Il lato concreto delle merci - che si tratti poi di beni oppure di servizi non fa differenza - diventa subordinato, è un mero portatore per l'unica cosa che conta in un'economia capitalista: la quantità di valore. II lato concreto, che è quello che corrisponde ai bisogni e desideri umani e dovrebbe costituire lo scopo della produzione viene invece nella società della merce ridotto a dipendere da quello che in verità ne deriva: la sua rappresentazione astratta.

Non si tratta di un'illusione o di una messa in scena, ma del livello più profondo, e ben reale, della logica capitalista. Mentre lo sfruttamento e la divisione in classi si trovano anche in molte altre società, questa inversione tra astratto e concreto è un tratto distintivo del solo capitalismo. Non si tratta tuttavia di un fenomeno "astratto" o secondario: esso spiega il carattere distruttivo e cieco della società capitalista e costituisce anche la vera radice della devastazione delle basi naturali della vita ad opera dell'economia 5 .

Questa lettura del feticismo ha guadagnato molto terreno negli ultimi decenni e ha sostituito largamente i concetti più problematici di "alienazione" (del giovane Marx) e di "reifıcazione" (di György Lukács). Ma quale nesso si può stabilire con il concetto di narcisismo?

E' stato introdotto da Freud a partire dal 1910. II narcisismo consiste essenzialmente in una indistinzione tra sé e il mondo circostante e nella tendenza a percepire il mondo esterno - gli "oggetti" in senso largo,

5 Per approfondire questi cenni rapidissimi non posso che rimandare al mio libro Le avventure della merce: per una critica del valore, Roma, Aracne Editrice, 2019. 
incluse le persone - solo come proiezioni e prolungamenti del proprio io. Si tratta di una fase necessaria nello sviluppo psichico del bambino molto piccolo che si difende dalla penosa percezione della sua impotenza reale creando una sensazione di fusione tra sé e il mondo che porta a dei sentimenti di onnipotenza. Problematico è invece il "narcisismo secondario" dell'adulto che non ha veramente superato la fase narcisista, il che avviene normalmente con il complesso d'Edipo in seguito al quale il bambino abbandona le sue fantasie di onnipotenza per sostituirle con delle soddisfazioni limitate, ma reali. Ma siccome questa rinuncia è dolorosa, delle tracce più o meno forti, e normalmente inconsce, del narcisismo originario possono continuare nella vita adulta e assumere tratti patologici. Il narcisista non è intimamente convinto della reale presenza di un mondo esterno con cui deve fare i conti, ma tenta di mettere tutto e tutti al suo servizio.

L'importanza del narcisismo come patologia è comunque limitata nell'opera di Freud, che è rivolta essenzialmente allo studio delle nevrosi. A partire degli anni settanta del Novecento, è aumentata fortemente la sua importanza nel discorso degli psicanalisti e degli psichiatri, che hanno ormai molto spesso a che fare con persone che soffrono di disturbi di tipo narcisista. Ma è stato lo statunitense Christopher Lasch che ha introdotto il narcisismo nel discorso sociologico e politico con il suo libro La cultura del narcisismo (1979). Secondo lui, la cultura contemporanea è profondamente marcata dal narcisismo. Soprattutto le fantasie di fusione e di onnipotenza si ritrovano in fenomeni cosi diversi come il ricorso alle tecnologie e il New Age, il disinteresse per la politica o l'arte minimalista. Il narcisismo non è dunque limitato a singoli individui patologici, ma impregna anche le scelte collettive.

Lasch ha però poco da dire sulle cause di un tale cambiamento epocale. Si pone perciò la necessità di pensare insieme il suo concetto di narcisismo, la cui fertilità pare indubbio, e una critica del capitalismo contemporaneo imperniata sui concetti di valore, di lavoro astratto e di feticismo della merce.

L'aumento del "tasso di narcisismo" nel corso del Novecento potrebbe essere legato alla diffusione della logica del valore e della merce in tutti i pori della società. Questa logica, con la sua inversione del concreto 
e dell'astratto, ha da tempo superato i confini della sola produzione di merci per invadere e colonizzare tutte le sfere della vita, soprattutto i consumi e i rapporti interpersonali. I mutamenti nella psiche degli individui sono allo stesso tempo causa e conseguenza dell'evoluzione nell'ambito produttivo che sarebbe molto riduttivo chiamare solo "economia": I'inversione tra astratto e concreto è una struttura di base che pervade, in mille forme, tutta la società moderna e le cui origini si possono rintracciare fino a Cartesio. Non c'è una priorità tra fattori "oggettivi" e "soggettivi", tra "base" e "sovrastruttura", tra "produzione materiale" e "coscienza". La classica nevrosi, che predominava ai tempi di Freud, era la conseguenza delle rinunce imposte agli individui che si obbligavano a dirigere le loro energie libidiche e aggressive verso il lavoro produttivo. Dagli anni sessanta in poi l'epicentro della vita sociale si è (apparentemente) spostato verso i consumi. L'ingiunzione fatta agli individui non era più: "Lavora, risparmia, sacrificati, obbedisci" (come prolungamento dell'"Ora et labora" della Chiesa), ma "Consuma, divertiti, spendi, indebitati". Invece di ricordare continuamente ai soggetti i loro limiti e la loro dipendenza da istanze superiori (Stato, religione, morale, gerarchie sociali), li si incitano a superare tutti i limiti (almeno nell'immaginazione) e a credere che il mondo sia a loro disposizione: il che è naturalmente una menzogna, ma permette di spingere a un consumo continuo di merci e di immagini che danno almeno l'impressione che sia così. Più il consumatore crede di poter avere "tutto" nella vita - e la pubblicità glielo ricorda almeno cento volte al giorno - più sarà disposto a lavorare come un folle e a indebitarsi per comprare le sue droghe (in senso letterale e traslato). E dunque a far girare la macchina sempre più affaticata della riproduzione capitalista.

Esiste poi un livello ancora più profondo del nesso tra narcisismo e feticismo. II narcisismo consiste in una svalutazione del mondo esterno al soggetto: tutti gli oggetti non sono in fondo che proiezioni del soggetto e non hanno una realtà propria, un'autonomia da rispettare. Questo soggetto è però da parte sua vuoto, perché non si è mai arricchito nei rapporti con gli oggetti esterni ed è piuttosto rimasto prigioniero dei suo fantasmi. Un individuo narcisista non è affatto forte, come si potrebbe credere, ma debole, perché è "senza mondo". Ora, questa assenza di mondo, questo diniego delle qualità diversificate del reale a favore di un'unica sostanza 
uguale (I'lo), che da parte sua è priva di qualità ed astratta, è ciò che caratterizza il narcisismo - ma ugualmente la logica del valore e del lavoro astratto. Per la logica del valore, tutto il mondo consta solo in porzioni di quantità prive di qualità intrinseca, cioè di merci che si distinguono solamente per la quantità di lavoro astratto che contengono. Si tratta di una svalutazione, di uno svuotamento del concreto - la molteplicità dell'esistente si trova ridotta a consistere solo in pezzi di una sostanza astratta sempre uguale (il valore). Per il narcisista, il mondo del non-io perde la sua realtà autonoma, per la logica feticista della merce, il mondo fuori dall'accumulazione di unità di lavoro astratto è solo un'ombra ${ }^{6}$. Ecco perché feticismo e narcisismo vanno ormai insieme. Per la nostra più grande disgrazia.

6 Anche qui, non posso fare que romandare, per un sviluppo oiù detagliato, al mio libro La société autophage: Capitalisme, démesure et autodestruction, Paris, La Découverte, 2017. 